\title{
Dynamics of below-the-knee arterial blood flow after endovascular revascularisation of peripheral arteries as a potential predictor of clinical outcomes during one-year follow-up
}

\author{
Paweł Kaczmarczyk ${ }^{1,2}$, Marek Krzanowski1, ${ }^{1,}$, Ewelina Szybiak ${ }^{3}$, Mikołaj Maga ${ }^{3}$, Agnieszka Wachsmann",

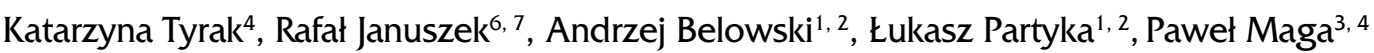 \\ 'Angio-Medicus Treatment Facility, Krakow, Poland \\ ${ }^{2}$ Department of Angiology, University Hospital, Krakow, Poland \\ 3Jagiellonian University Medical College, Krakow, Poland \\ ${ }^{4}$ Department of Angiology, Jagiellonian University Medical College, Krakow, Poland \\ ${ }^{5}$ Department of Dermatology, Jagiellonian University Medical College, Krakow, Poland \\ ${ }^{6}$ Department of Clinical Rehabilitation, University of Physical Education, Krakow, Poland \\ ${ }^{72}$ nd Department of Cardiology and Cardiovascular Interventions, University Hospital, Krakow, Poland
}

\begin{abstract}
Background: Patients with advanced lower limb ischaemia are, at present, mainly treated using revascularisation.

Aim: The aim of the study was to investigate whether the dynamics of blood flow in below-the-knee (BTK) arteries assessed by angiography correlate with clinical outcomes after a 12-month follow-up in patients with severe leg ischaemia treated percutaneously.

Methods: The current study enrolled 287 consecutive patients who underwent 302 endovascular procedures on the infrainguinal arteries. The mean age of the included participants was $67.4 \pm 10.4$ years. After the procedure, blood flow in all patent BTK arteries was assessed using frame count (FC). Patients were then evaluated after one, three, six, and 12 months. During the follow-up visits, clinical condition was evaluated based on the Rutherford scale, ankle-brachial index, and the need for reintervention or amputation.

Results: Clinical improvement at the end of the follow-up period was observed in $242(80.1 \%)$ cases and no improvement or worsening in was seen in $42(13.0 \%)$ patients. In total, $66(21.8 \%)$ reinterventions and $18(6 \%)$ amputations during the follow-up period were recorded. Patients with higher FC in the tibial anterior artery experienced significantly better clinical improvement within the 12-month follow-up period $(p=0.02)$. Lower FC predisposed to worse clinical outcomes after angioplasty. Similar tendencies were found for the tibial posterior and fibular arteries but without statistical significance.

Conclusions: The results suggest a negative relationship between FC observed on the final angiogram and clinical outcomes in patients undergoing endovascular treatment of the peripheral arteries.
\end{abstract}

Key words: clinical endpoints, endovascular treatment, frame count, peripheral arterial disease, predictors

Kardiol Pol 2019; 77, 1: 24-32

\section{INTRODUCTION}

Patients with advanced lower limb ischaemia are, at present, mainly treated using revascularisation. While percutaneous treatment is becoming increasingly popular due to lower periprocedural risks compared with open vascular surgery, it seems that the long-term outcomes of any type of revascularisation are far from optimal [1]. Recently, significant improvement of devices used for endovascular treatment

Address for correspondence:

Rafał Januszek, MD, PhD, $2^{\text {nd }}$ Department of Cardiology and Cardiovascular Interventions, University Hospital in Krakow, ul. Kopernika 17, 31-501 Kraków, Poland, tel: +48 1242471 70, fax: +48 1242471 80, e-mail: jaanraf@interia.pl

Received: 28.07.2018 Accepted: 5.10.2018 Available as AoP: 8.10.2018

Kardiologia Polska Copyright (C) Polish Cardiac Society 2019 
and techniques of revascularisation have been introduced. The development of a system that would be able to predict clinical outcomes in patients with peripheral arterial disease (PAD), such as amputation-free survival or overall survival, could help clinicians in decision-making and guiding the appropriate usage of health care resources. To date, a number of studies have been published demonstrating predictors of clinical outcome in patients after percutaneous transluminal angioplasty (PTA) of the infrainguinal arteries [2, 3]. Among others, biochemical indices such as the urinary leukotriene E4 or thromboxane were found to be associated with long-term results [4]. Apart from biomarkers and clinical scoring systems, clinical outcomes could also be predicted based on angiographic findings, including the runoff grade [5], which was designed to evaluate treatment results after bypass surgery. Despite its subjective form, it was proven to predict clinical outcome [6, 7]. There are scoring systems used in cardiology that can predict clinical outcomes in patients who have undergone percutaneous coronary interventions. The Thrombolysis In Myocardial Infarction (TIMI) flow grade and the corrected TIMI frame count (FC) are among the most commonly used methods. Despite their subjectivity, they are simple, easy-to-use, and time-effective tools to assess the dynamics of blood flow in the epicardial coronary arteries $[8,9]$. However, the currently used scoring systems are not sufficient, and there is still a need to create more objective ones dedicated to the peripheral vessels, which could predict immediate and long-term clinical outcomes.

Therefore, the aim of the current study was to investigate whether the dynamics of blood flow in below-the-knee (BTK) arteries assessed by angiography are associated with clinical outcome during a 12-month follow-up among patients with severe leg ischaemia treated percutaneously.

\section{METHODS}

\section{Study population, design, and definitions}

The current study enrolled 287 consecutive patients who underwent 302 endovascular treatments on the infrainguinal arteries at a large angiology centre. The patient recruitment lasted from March until October 2015. The mean age of the included participants was $67.4 \pm 10.4$ years (Table 1 ). There were 162 procedures performed in critical limb ischaemia (CLI) patients and 140 procedures in patients with limb claudication (non-CLI patients) (Table 2). After the procedure, blood flow in all patent BTK arteries was assessed using the FC evaluation method adapted to the purposes of the present study. Patients were then evaluated after one, three, six, and 12 months. During the follow-up visits, clinical condition was assessed based on the Rutherford scale, ankle-brachial index $(\mathrm{ABI})$, as well as reintervention and amputation rates. Medical therapy after percutaneous interventions was continued according to the current recommendations. Chronic kidney disease was defined as impaired kidney function expressed as decreased glomerular filtration rate $\left(<60 \mathrm{~mL} / \mathrm{min} / 1.73 \mathrm{~m}^{2}\right)$ for three months or more, irrespective of the cause, estimated using the Cockcroft-Gault formula. Hypercholesterolaemia, hypertension, diabetes, coronary artery disease, and heart failure were defined according to the European Society of Cardiology guidelines and previous diagnoses from prior hospital discharge cards [10-14]. Other diagnoses were taken into account on the basis of the information found on prior hospital discharge cards. The study complies with the Declaration of Helsinki. All subjects provided written and informed consent for participation prior to enrolment.

\section{Frame count}

Blood flow rate in the BTK arteries was assessed by means of digital subtraction angiography using the Integris $\vee 3000$ angiography unit (Philips, Eindhoven, Netherlands). Patients were examined in a supine position. The source-to-image distance was kept at a constant of $100 \mathrm{~cm}$. A 31-cm field of view diameter was used, and the image intensifier was positioned against the calf of the patient. The intensifier was positioned in such a way that the maximal length of the calf was visualised, with intercondylar prominence of the tibia maintained at the uppermost point of the image. The contrast medium (Ultravist 370, Schering Pharma AG, Berlin, Germany) was manually injected into the sheath positioned into the ipsilateral common femoral artery. Images were recorded at a rate of six frames per second. The number of frames between the first arrival of the contrast at the proximal part of the image and its reaching the most distal part of each of the axial calf arteries, i.e. the tibial arteries, and the fibular artery (FA), was measured for each of the vessels and used for further analysis. The first frame was defined as the one in which the contrast dye fully entered the artery, fulfilling all of the following criteria: a) a column of fully concentrated dye had to be extended across the entire width of the origin of the artery, b) the dye had to touch both borders of the origin of the artery, and c) there had to be antegrade motion of the dye. The last frame was defined as the one in which the dye first entered the artery at the distal edge of the image. Full opacification of the artery was not required. Often, the final frame is best determined by running the cine film past the initial opacification of the end-point branch and then moving frame-by-frame in reverse until the endpoint branch disappears [9]. We did not correct the BTK FC for the length of the arteries because the purpose of the study was to assess the blood flow velocity in the BTK arteries, regardless of the length of the vessels that supply blood to them, and consequently, the height and surface area of the patients.

\section{Statistical analysis}

The schedule frequency characteristics were measured on a nominal or ordinal scale and, for continuous variables, by using descriptive statistics including arithmetic mean 
Table 1. General characteristics of the patients

\begin{tabular}{lcccc} 
& $\begin{array}{c}\text { Overall group } \\
(\mathbf{n}=\mathbf{3 0 2})\end{array}$ & $\begin{array}{c}\text { CLI } \\
(\mathbf{n}=\mathbf{1 6 2})\end{array}$ & $\begin{array}{c}\text { Non-CLI } \\
(\mathbf{n}=\mathbf{1 4 0 )}\end{array}$ \\
\hline Male/female sex & $180 / 122$ & $90 / 72$ & $92 / 48$ & 0.07 \\
Age [years] & $67.4 \pm 10.4$ & $69.4 \pm 10.2$ & $65.0 \pm 10.1$ & $<0.001$ \\
Current smokers & $52(17.2)$ & $29(17.9)$ & $23(16.4)$ & 0.69 \\
Former smokers & $119(39.4)$ & $56(34.5)$ & $63(45)$ & 0.07 \\
Prior cerebral stroke/TIA & $32(10.6)$ & $21(13.0)$ & $11(7.8)$ & 0.14 \\
Glomerular filtration rate $\left[\mathrm{mL} / \mathrm{min} / \mathrm{m}^{2}\right]$ & $72.3 \pm 24.3$ & $69.4 \pm 25.7$ & $75.7 \pm 22.1$ & 0.02 \\
Kidney failure & $39(12.9)$ & $26(16.0)$ & $13(9.3)$ & 0.07 \\
Dialysis therapy & $6(1.9)$ & $5(3.1)$ & $1(0.7)$ & 0.13 \\
Heart failure & $25(8.3)$ & $19(11.7)$ & $6(4.3)$ & 0.01 \\
Coronary artery disease & $142(47.0)$ & $77(47.5)$ & $65(46.4)$ & 0.76 \\
Prior myocardial infarction & $56(18.5)$ & $31(19.1)$ & $25(17.8)$ & 0.73 \\
Hypertension & $232(76.8)$ & $124(76.5)$ & $108(77.1)$ & 0.94 \\
Diabetes & $143(47.3)$ & $92(56.8)$ & $51(36.4)$ & $<0.001$ \\
Insulin therapy & $96(31.8)$ & $67(41.3)$ & $29(20.7)$ & $<0.001$ \\
Hypercholesterolaemia & $85(28.1)$ & $48(29.6)$ & $37(26.4)$ & 0.49 \\
\hline
\end{tabular}

Data are presented as arithmetic mean \pm standard deviation or number (percentage). CLI — critical limb ischaemia; TIA — transient ischaemic attack

and standard deviation or median and interquartile range, where applicable. Normal distribution was checked with the Shapiro-Wilk test. Student t-test or the Mann-Whitney U test was used when applicable for the comparison of continuous variables, while the $\chi^{2}$ test was applied for categorical variables. ANOVA analysis was used to assess trend significance at consecutive follow-up visits. A model based on the retrograde correction method was constructed for the purpose of performing a multivariate analysis. The model included anthropometric indices, concomitant diseases, smoking status, age, sex, kidney function parameters, clinical presentation of PAD at baseline, location of the culprit artery and PTA, number of occluded arteries, $\mathrm{ABI}$, and Rutherford class at baseline. Receiver operator characteristic (ROC) curve analysis was performed to determine optimal cut-off values when the sensitivity approximates specificity, as well as their relationship between TIMI FC and study endpoints. A p-value of $<0.05$ was considered statistically significant. Due to the fact that the results of the current study were not a primary outcome, we did not calculate the power of the study. However, in a power analysis performed after the study for the alpha coefficient of $2.5 \%$ and almost 100 patients with a composite study endpoint and 200 patients without it, the power was calculated at $80 \%$, which is a standard result and should be sufficient to determine the relationships between particular factors in the two groups. Statistical analyses were performed using Statistica 10.0 software (Dell Software, Inc., Round Rock, TX, USA) and SPSS STATISTICS 24 (IBM, Armonk, NY, USA).

\section{RESULTS}

Clinical characteristics of all participants (Table 1), characteristics of the PTA procedures, including lesion location and type (stenosis vs. occlusion), number of occluded BTK and pedal arteries, as well as the FC (Table 2) showed differences between the CLI and non-CLI patients. Clinical improvement at the end of the follow-up period was observed in a total of $242(80.1 \%)$ cases, and no improvement or worsening was seen in $42(13.0 \%)$ cases (Figs. 1, 2). Altogether, 66 (21.8\%) reinterventions and 18 (6\%) amputations were recorded during the follow-up period (Table 3 ). In the group of patients that required reinterventions, $40(60.6 \%)$ cases improved clinically, 13 (19.7\%) presented stable clinical symptoms, three (4.5\%) patients worsened clinically, and 10 (15.5\%) underwent amputation. In the group of patients without reinterventions (236 procedures; $78.1 \%$ ) clinical improvement was noted in $202(85.6 \%)$ cases, no improvement in $25(10.6 \%)$, worsening in one $(0.4 \%)$, and amputation in eight $(3.4 \%)$. The endpoint was observed in a lower number of patients $(n=16)$ : three patients died during the follow-up period and 13 were lost to follow-up due to unspecified reasons. While assessing the distribution of FC in particular BTK arteries, most patients represented the groups with FC lower than six, between five and 10, and between 10 and 20 (Fig. 2).

\section{Dynamics of blood flow}

The dynamics of blood flow were assessed in the anterior tibial artery (ATA), posterior tibial artery (TPA), and FA. Higher FC (slower blood flow) was found in younger patients (ATA: 
Table 2. Atherosclerotic lesion characteristics and frame count

\begin{tabular}{|c|c|c|c|c|}
\hline & $\begin{array}{l}\text { Overall group } \\
\qquad(\mathrm{n}=302)\end{array}$ & $\begin{array}{c}\text { CLI } \\
(n=162)\end{array}$ & $\begin{array}{l}\text { Non-CLI } \\
(n=140)\end{array}$ & p \\
\hline \multicolumn{5}{|l|}{ Level of PTA: } \\
\hline Superficial femoral artery & $215(71.2)$ & $97(59.9)$ & $118(84.3)$ & $<0.001$ \\
\hline Popliteal artery & $130(43.0)$ & $78(48.1)$ & $52(37.1)$ & 0.054 \\
\hline BTK artery & $148(49.0)$ & $112(69.1)$ & $36(25.7)$ & $<0.001$ \\
\hline \multicolumn{5}{|l|}{ Occluded BTK arteries: } \\
\hline None & $55(18.2)$ & $24(14.8)$ & $31(22.1)$ & 0.09 \\
\hline One & $158(52.3)$ & $78(48.1)$ & $80(57.1)$ & 0.0004 \\
\hline Two & $89(29.4)$ & $60(37.0)$ & $29(20.7)$ & 0.002 \\
\hline \multicolumn{5}{|l|}{ Lesion type before PTA: } \\
\hline Stenosis & $114(37.7)$ & $48(29.6)$ & $66(47.1)$ & 0.0017 \\
\hline Occlusion & $188(62.2)$ & $114(70.4)$ & $74(52.8)$ & 0.0017 \\
\hline \multicolumn{5}{|l|}{ Occluded foot arteries: } \\
\hline None & $89(29.5)$ & $40(24.7)$ & $49(35)$ & 0.05 \\
\hline One & $142(47.0)$ & $77(47.5)$ & $65(46.4)$ & 0.84 \\
\hline Two & $71(23.5)$ & $45(27.8)$ & $26(18.6)$ & 0.059 \\
\hline \multicolumn{5}{|l|}{ TASC classification: } \\
\hline 0 & $42(13.9)$ & $36(22.2)$ & $6(4.3)$ & 0.039 \\
\hline A & $37(12.2)$ & $14(8.6)$ & $23(16.4)$ & $<0.001$ \\
\hline B & $76(25.1)$ & $20(12.3)$ & $56(40)$ & $<0.001$ \\
\hline C & $64(21.2)$ & $33(20.4)$ & $31(22.1)$ & 0.7 \\
\hline $\mathrm{D}$ & $83(27.5)$ & $59(34.4)$ & $24(17.1)$ & 0.0002 \\
\hline \multicolumn{5}{|c|}{ Frame count (frames per artery): } \\
\hline \multirow[t]{2}{*}{ Anterior tibial artery } & $11.3 \pm 12.1$ & $8.0 \pm 7.7$ & $14.4 \pm 14.4$ & $<0.001$ \\
\hline & $8(5 \div 12,7)$ & $6(4 \div 9)$ & $11(6 \div 17)$ & \\
\hline \multirow[t]{2}{*}{ Tibial posterior artery } & $11.3 \pm 10.0$ & $9.1 \pm 7.8$ & $13.4 \pm 11.3$ & 0.009 \\
\hline & $8(5 \div 15)$ & $6(4.2 \div 10)$ & $10(5.5 \div 17)$ & \\
\hline \multirow[t]{2}{*}{ Fibular artery } & $11.7 \pm 8.3$ & $8.8 \pm 4.9$ & $15.1 \pm 10.0$ & $<0.001$ \\
\hline & $9(6 \div 14.2)$ & $7(6 \div 11)$ & $12(8 \div 20)$ & \\
\hline
\end{tabular}

Data are presented as arithmetic mean \pm standard deviation, median $\div$ interquartile range, or number (percentage). BTK - below-the-knee; CLI — critical limb ischaemia; PTA — percutaneous transluminal angioplasty; TASC — Trans-Atlantic Inter-Society Consensus Document on Management of Peripheral Arterial Disease

$p=0.052, r=-0.13 ;$ FA: $p=0.02, r=-0.15)$ and in those with less advanced clinical symptoms, i.e. lower Rutherford class (ATA: $p<0.001, r=-0.28$; TPA: $p=0.001, r=-0.21$; FA: $p<0.001, r=-0.38)$. Similar observations were made for patients with less advanced angiological findings such as lower Trans-Atlantic Inter-Society Consensus Document on Management of Peripheral Arterial Disease (TASC) grade (ATA: $p=0.01, r=-0.16$; TPA: $p=0.01, r=-0.2 ;$ FA: $p=0.01$, $r=-0.16$ ) and a larger number of patent BTK vessels (ATA: $p=0.01, r=-0.17 ;$ FA: $p<0.0001, r=-0.28)$. We found that FC was higher in non-CLI patients (ATA: $p<0.0001$, $r=-0.26$; TPA: $p=0.009, r=-0.21$; FA: $p<0.0001$, $r=-0.37$ ), those who underwent treatment of the super- ficial femoral artery in comparison to other locations (ATA: $p=0.03, r=0.14)$, and in patients with higher mean $A B I$ value (TPA: $p=0.001, r=0.25$ ). This trend was similar when the groups of patients with CLI and those with claudication were analysed separately. The relationship between the level of PTA, clinical study endpoints, and FC according to particular BTK arteries is presented in Table 4.

\section{Frame count and clinical outcomes}

Patients with higher FC (slower blood flow) in ATA had a significantly greater clinical improvement (based on the Rutherford scale) during the 12-month follow-up period ( $p=0.02$ ). Lower FC (faster blood flow) predisposed to worse clinical 

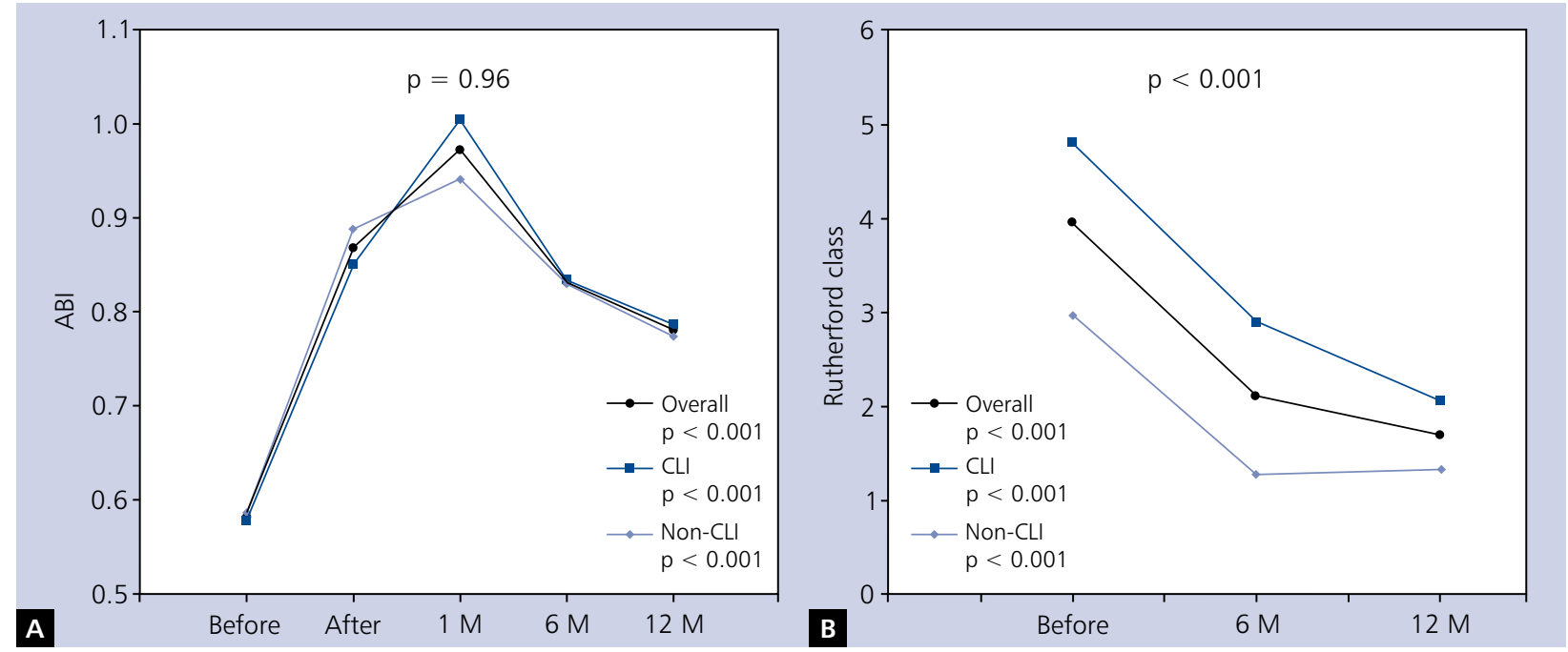

Figure 1. A. Mean values of the ankle-brachial index (ABI) at consecutive follow-up visits; B. Mean values of the Rutherford class at consecutive follow-up visits; CLI — critical limb ischaemia; $\mathrm{M}$ - months

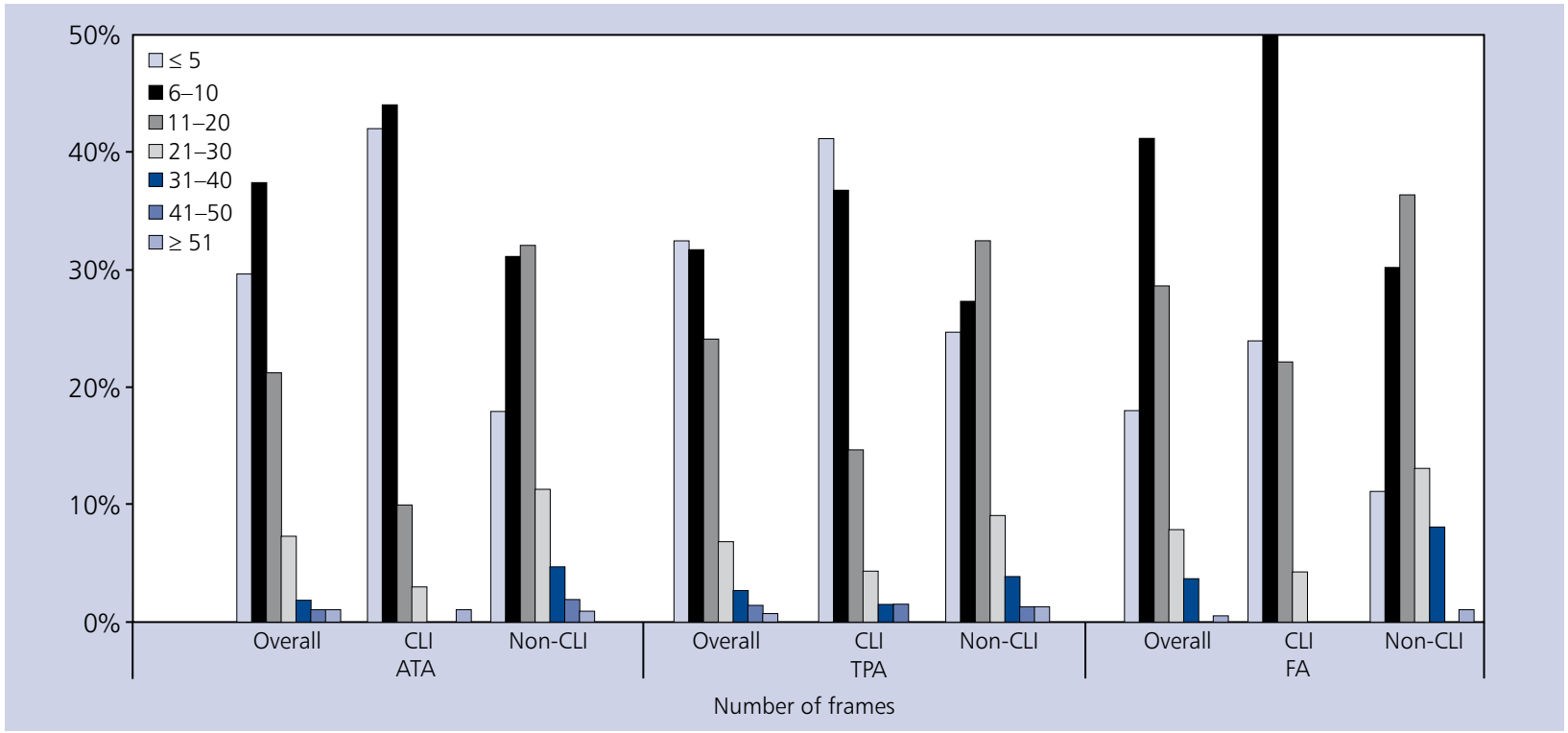

Figure 2. Quantitative distribution of the number of patients depending on frame count range; ATA — anterior tibial artery; CLI — critical limb ischaemia; FA — fibular artery; TPA — posterior tibial artery

outcomes after PTA (Fig. 3A). Similar tendencies were found for TPA and FA but without statistical significance.

\section{Frame count and ankle-brachial index}

We found no relationship between FC in particular BTK arteries and $\mathrm{ABI}$ (calculated as changes in $\mathrm{ABI}$ values measured directly before and after the procedure, as well as the $\mathrm{ABI}$ value after 12 months of follow-up and at baseline). There was a significant improvement of $\mathrm{ABI}$ values after PTA at consecutive follow-up visits ( $p<0.001)$ (Fig. 1A).

\section{Study endpoints}

The overview of the clinical study endpoints is presented in Tables 3 and 4.

\section{Amputations}

We found no relationship between the FC in the BTK arteries (ATA, TPA, and FA) and the number of amputations. The amputation rate during the follow-up period was almost two times greater in patients with occluded ATA after PTA compared to those with patent ATA, but without statistical 
Table 3. Study endpoints after 12 months of follow-up

\begin{tabular}{lcccc|} 
& $\begin{array}{c}\text { Overall group } \\
(\mathbf{n}=\mathbf{3 0 2})\end{array}$ & $\begin{array}{c}\text { CLI } \\
(\mathbf{n}=162)\end{array}$ & $\begin{array}{c}\text { Non-CLI } \\
(\mathbf{n}=140)\end{array}$ & 0.003 \\
\hline Reinterventions & $66(21.8)$ & $46(28.4)$ & $20(14.3)$ & $<0.001$ \\
Amputations & $18(6.0)$ & $17(10.5)$ & $1(0.7)$ & 0.41 \\
No clinical improvement & $60(19.9)$ & $35(21.6)$ & $25(17.8)$ & 0.03 \\
Composite study endpoint & $98(32.4)$ & $61(37.6)$ & $37(26.4)$ & \\
\hline
\end{tabular}

Data are presented as number (percentage). No clinical improvement means that after 12 months of follow-up, the Rutherford class was the same or worse than before percutaneous transluminal angioplasty, with the inclusion of patients with amputation. CLI — critical limb ischaemia

Table 4. Study endpoints after 12 months of follow-up and frame count according to the location of the treated lesion

\begin{tabular}{|c|c|c|c|c|}
\hline & $\begin{array}{l}\text { Superficial femoral } \\
\text { artery }(n=215)\end{array}$ & $\begin{array}{l}\text { Popliteal artery } \\
\qquad(n=130)\end{array}$ & $\begin{array}{l}\text { Below-the-knee } \\
\text { artery }(n=148)\end{array}$ & $p$ \\
\hline Reinterventions & $43(20)$ & $35(26.9)$ & $42(28.4)$ & 0.13 \\
\hline Amputations & $7(3.2)$ & $8(6.1)$ & $17(11.5)$ & 0.007 \\
\hline No clinical improvement & $34(15.8)$ & $33(25.4)$ & $40(27)$ & 0.02 \\
\hline Composite study end-point & $63(29.3)$ & $52(40)$ & $60(40.5)$ & 0.04 \\
\hline \multicolumn{5}{|l|}{ Frame count (frames per artery): } \\
\hline \multirow[t]{2}{*}{ Anterior tibial artery } & $12.5 \pm 13.7$ & $8.1 \pm 5.8$ & $9.5 \pm 8.9$ & 0.002 \\
\hline & $8(5 \div 14.7)$ & $6(4 \div 10)$ & $7(4 \div 10)$ & \\
\hline \multirow[t]{2}{*}{ Tibial posterior artery } & $11.9 \pm 10.4$ & $8.7 \pm 6.6$ & $9.9 \pm 7.9$ & 0.051 \\
\hline & $9(5 \div 15.7)$ & $6(5 \div 10)$ & $7(5 \div 12)$ & \\
\hline \multirow[t]{2}{*}{ Fibular artery } & $12.3 \pm 8.8$ & $9.2 \pm 5.8$ & $10.5 \pm 7.3$ & 0.06 \\
\hline & $9.5(6 \div 17)$ & $7(5 \div 11)$ & $8(6 \div 12)$ & \\
\hline
\end{tabular}

Data are presented as arithmetic mean \pm standard deviation, median $\div$ interquartile range, or number (percentage). No clinical improvement means that after 12 months of follow-up, the Rutherford class was the same or worse than before percutaneous transluminal angioplasty, with the inclusion of patients with amputation; clinical improvement means that after 12 months of follow-up, the Rutherford class has improved (decreased).

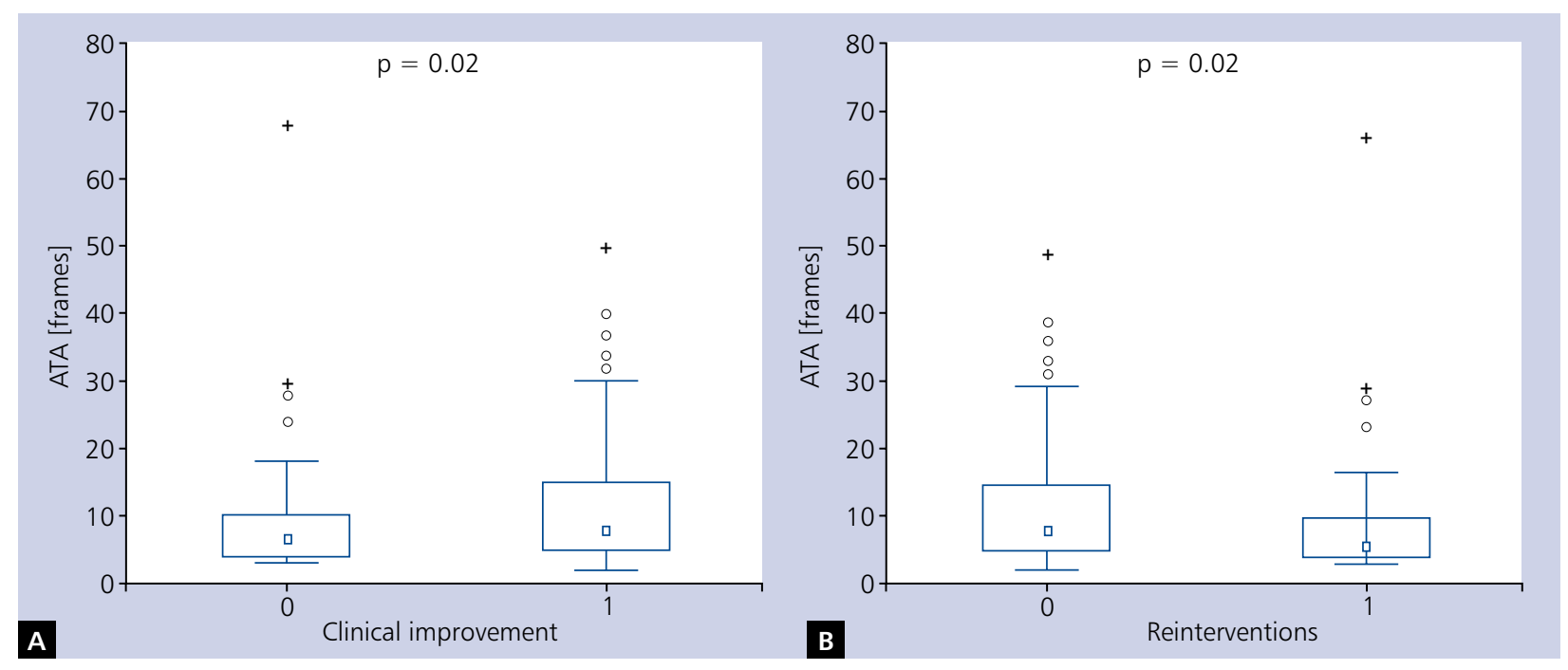

Figure 3. A. The relationship between frame count in anterior tibial artery (ATA) and clinical outcomes in patients with clinical improvement and stable clinical symptoms during the follow-up period vs. individuals with worse clinical symptoms, reinterventions, and amputations; B. The relationship between frame count in ATA and reinterventions in patients with clinical improvement and stable clinical symptoms during the follow-up period vs. individuals with worse clinical symptoms, reinterventions, and amputations 
significance $(8.33 \%$ vs. $4.85 \%, p=0.23)$. There were no correlations between the ATA, TPA, and FA. The amputation rate in patients with at least two occluded BTK arteries was higher compared to those in whom one artery was occluded or those without occlusion, but not significantly $(7.78 \%$ vs. $5.66 \%$ and $3.77 \%, p=0.6)$. The amputation rate was more than twofold higher in patients with occluded BTK arteries undergoing PTA compared to those with stenosed arteries, but without statistical significance $(7.45 \%$ vs. $3.51 \%, p=0.16)$.

\section{Reinterventions}

The amputation rate was higher among patients requiring reinterventions during the follow-up period compared to others $(15.1 \%$ vs. $3.3 \%, p<0.001)$. Patients with lower FC in ATA presented higher reintervention rates during the follow-up period (11.8 \pm 12.4 vs. $9.4 \pm 10.6$ frames, $p=0.02$; Fig. $3 \mathrm{~B}$ ). This relationship was not significant for TPA or FA. Occlusion of ATA, TPA, and FA after PTA was not related to a higher reintervention rate during the follow-up period. The percentage of reinterventions was not connected with the number of occluded arteries or the lesion type.

\section{CLI vs. non-CLI patients}

Patients with CLI were associated with a significantly lower number of FCs (faster blood flow) when compared to claudicants. Patients who improved or had stable symptoms after PTA within the 12 months of follow-up, those initially non-CLI, achieved better clinical outcomes $(p=0.005)$. The amputation rate during the 12-month follow-up period was significantly higher in CLI patients. Based on the Rutherford scale (Rutherford class from 2 to 6 ), the amputation rates were $0 \%, 0.7 \%, 2.1 \%, 11.8 \%$, and $26.6 \%$, respectively $(\mathrm{p}<0.001$ ). Similarly, the reintervention rate was the highest in CLI patients compared to non-CLI ones ( $p=0.03$ ).

\section{Multivariate and ROC analysis}

Multivariate analysis revealed that PTA performed on the popliteal artery was an independent predictor of better clinical outcome after 12 months of follow-up, expressed as a combination of amputation rate and lack of clinical improvement, in comparison to PTA of other infrainguinal arteries (hazard ratio $[\mathrm{HR}]=0.46$; 95\% confidence interval $[\mathrm{Cl}]$ 0.23-0.89; $\mathrm{p}=0.02$ ), while PTA of the BTK arteries was an independent predictor of increased amputation $(\mathrm{HR}=7.4 ; 95 \% \mathrm{Cl}$ 2.1-25.6; $p=0.002)$ and reintervention rates $(H R=2.1$; $95 \% \mathrm{Cl} 1.2-3.7 ; \mathrm{p}=0.008)$. In comparison to other locations, PTA of the popliteal $(\mathrm{HR}=1.8 ; 95 \% \mathrm{Cl} 1.1-2.9 ; \mathrm{p}=0.01)$ and $\mathrm{BTK}$ arteries $(\mathrm{HR}=1.9 ; 95 \% \mathrm{Cl} 1.2-3.2 ; \mathrm{p}=0.008)$ was found to be an independent predictor of the lack of clinical improvement accompanied by the need for reinterventions. The ROC curves assessing the relationships between FC measured in the BTK arteries after PTA did not meet the study endpoints. Our attempts to determine the cut-off points for FC that best display the risk of poorer clinical outcomes failed.

\section{DISCUSSION}

During the previous decade, angioplasty and stent placement became an accepted, and in many situations, preferred alternative to surgical treatment in patients with symptomatic limb ischaemia [1]. Despite their safety and effectiveness, the number of reinterventions related to endovascular procedures remains high. In the femoropopliteal region, restenosis rates in some groups of patients following endovascular procedures are described as higher than after a traditional bypass surgery [15]. Thus, improvement of patency is one of the major challenges for endovascular procedures.

In the past few years, many studies have been conducted focusing on factors that could influence the clinical outcome of endovascular treatment. Based on the BASIL study, factors that contributed to the survival model were age, presence of tissue loss, serum creatinine, $\mathrm{ABI}$, prior myocardial infarction, history of stroke, body mass index, smoking status, and Bollinger angiogram score [2]. The FINNVASC and PREVENT III studies evaluated the post-procedural risk level based on variables such as age, tissue loss, coronary artery disease, diabetes mellitus, or dialysis [3]. It was confirmed that haemodialysis alongside cilostazol administration, stent fracture, and TASC lesion type were independent predictors of successful femoropopliteal stenting with nitinol stents [16]. Research performed among patients with end-stage kidney disease and their coronary arteries revealed a reduction in blood flow velocity assessed using the FC method [17]. Based on this, it was concluded that some comorbidities inducing decreased blood flow in the arteries could impact clinical outcomes following PTA of the peripheral arteries. There have been many reports stating that vessel runoff could be a strong predictor of clinical outcomes after endovascular treatment [7, 18, 19]. Conventionally, evaluation of vessel runoff has been estimated based on the Society for Vascular Surgery (SVS) runoff score or the number of patent tibial vessels [18]. The SVS runoff score was, in fact, an effective tool in predicting treatment outcomes, but its complexity in the evaluation process made it too sophisticated for daily medical practice. There are some reports stating that the SVS runoff score was not reproducible among observers [20]. Based on the experience taken from interventional cardiology, the idea of using flow grades as a predictor of clinical outcomes in patients after endovascular procedures on the peripheral vessels was introduced. Hiramori et al. [6] evaluated the runoff grade by the final angiogram after endovascular therapy. Also, Davies et al. [7] proved that in patients presenting resting pain and tissue loss treated with endovascular techniques, patency was negatively affected by compromised and poor runoffs. Although the runoff grade was shown to be an independent predictor 
of endovascular treatment outcome, opinions were still based on the subjective evaluation of vessel blood flow, making it vulnerable to potential errors [21]. Therefore, in the current study, we aimed to investigate the relationship between the number of frames counted in the final angiogram and factors that may influence the clinical outcome after endovascular treatment. Statistically significant correlations have been found between the number of counted frames for the BTK arteries and age, Rutherford class, number of occluded BTK arteries, TASC classification, level of treated vessel, and the presence of critical limb ischaemia. Also, slower blood flow in the calf vessels was generally detected among the healthier population. Multiple studies have proven that improvement of clinical symptoms is related to the acceleration of peripheral blood flow after endovascular treatment compared to preoperative condition [22]. It has also already been stated that patients without significant blood flow after endovascular treatment have worse cumulative outcomes [6]. Salapura et al. [23] stated that faster blood flow after endovascular treatment had better prognostic value only within the first month of observation. He found that clinical improvement in the 12-month follow-up was significantly related to slower blood flow in the calf arteries. What is more, the lower FC (faster blood flow) predisposed to worse clinical outcomes after PTA. These finding were observed most significantly with regard to the anterior tibial artery, but the same phenomena were also present for TPA and FA. The current study demonstrated that PTA performed on the popliteal and BTK arteries is a prognostic factor of poorer clinical outcomes during follow-up. This was not significantly related to the FC, despite the fact that the relationship between FC and the PTA level was initially observed. Furthermore, univariate analysis also showed such a relationship, which turned out to be statistically significant in the case of amputation, the lack of clinical improvement, and composite study endpoints. However, the relationship between FC, PTA, and clinical outcomes proved to be too weak to be confirmed using multivariate analysis. Based on magnetic resonance angiography, Prince et al. [24] found that female patients with type 2 diabetes mellitus and cellulitis or ulcerations on the legs tended to have faster blood flow in the lower limb arteries. It has been hypothesised that faster blood flow is a result of vascular calcifications and decreased arterial compliance, which forces faster flow velocity. More advanced clinical condition (higher Rutherford class) is frequently related to the presence of peripheral ulcers that can result in vasodilatation and increased demand for flow, thereby causing its acceleration. Another possible explanation for the presence of faster blood flow in patients with more advanced PAD is the fact that with peripheral vessel occlusions, the velocity of blood flow can be related to the smaller cross-sectional diameter of collateral vessels compared to the main arteries. It may also relate to distal ischaemia, which stimulates vasodilation distal to occlusions. The FC technique presented in the current study is a simple and objective method created to predict clinical outcomes after endovascular procedures on the peripheral arteries.

The FC could be influenced by additional factors such as coronary perfusion pressure (including the severity of heart failure expressed as left ventricular ejection fraction), pressure of the contrast injection, vessel size, the length of lower limb arteries above the BTK arteries, the number of branches, stenosis severity, heart rate (including atrial fibrillation), the use of certain medications, and other factors related to endothelial function and the coagulation system.

In conclusion, the results suggest a negative relationship between FC observed on the final angiogram and clinical outcomes in patients undergoing endovascular treatment of the peripheral arteries. FC should be considered as a simple and effective index that can be used in the future to predict vessel patency in this group of patients.

\section{Conflict of interest: none declared}

\section{References}

1. SetacciC, de Donato G, Teraa M, et al. Chapter IV: Treatment of critical limb ischaemia. Eur J Vasc Endovasc Surg. 2011; 42 (Suppl 2): S43-S59, doi: 10.1016/S1078-5884(11)60014-2, indexed in Pubmed: 22172473.

2. Moxey PW, Brownrigg J, Kumar SS, et al. The BASIL survival prediction model in patients with peripheral arterial disease undergoing revascularization in a university hospital setting and comparison with the FINNVASC and modified PREVENT scores. J Vasc Surg. 2013; 57(1): 1-7, doi: 10.1016/j. jvs.2012.04.074, indexed in Pubmed: 23040797.

3. Arvela E, Söderström M, Korhonen M, et al. Finnvasc score and modified Prevent III score predict long-term outcome after infrainguinal surgical and endovascular revascularization for critical limb ischemia. J Vasc Surg. 2010; 52(5): 1218-1225, doi: 10.1016/j.jvs.2010.06.101, indexed in Pubmed: 20709482.

4. Maga P, Sanak M, Jawien J, et al. 11-dehydro thromboxane B2 levels after percutaneous transluminal angioplasty in patients with peripheral arterial occlusive disease during a one year follow-up period. J Physiol Pharmacol. 2016; 67(3): 377-383, indexed in Pubmed: 27511998.

5. Davies AH, Magee TR, Parry R, et al. Evaluation of distal run-off before femorodistal bypass. Cardiovasc Surg. 1996; 4(2): 161-164, indexed in Pubmed: 8861429.

6. Hiramori S, Soga Y, Tomoi Y, et al. Impact of runoff grade after endovascular therapy for femoropopliteal lesions. J Vasc Surg. 2014; 59(3): 720-727, doi: 10.1016/j.jvs.2013.09.053, indexed in Pubmed: 24377941.

7. Davies MG, Saad WE, Peden EK, et al. Impact of runoff on superficial femoral artery endoluminal interventions for rest pain and tissue loss. J Vasc Surg. 2008; 48(3): 619-625, doi: 10.1016/j. jvs.2008.04.013, indexed in Pubmed: 18727964.

8. Perez de Prado A, Fernández-Vázquez F, Cuellas-Ramón JC, et al. Coronary clearance frame count: a new index of microvascular perfusion. J Thromb Thrombolysis. 2005; 19(2): 97-100, doi: 10.1007/s11239-005-1379-5, indexed in Pubmed: 16052299.

9. Gibson CM, Cannon CP, Daley WL, et al. TIMI frame count: a quantitative method of assessing coronary artery flow. Circulation. 1996; 93(5): 879-888, indexed in Pubmed: 8598078. 
10. Reiner Z, Catapano AL, De Backer G, et al. European Association for Cardiovascular Prevention \& Rehabilitation, ESC Committee for Practice Guidelines (CPG) 2008-2010 and 2010-2012 Committees. ESC/EAS Guidelines for the management of dyslipidaemias: the Task Force for the management of dyslipidaemias of the European Society of Cardiology (ESC) and the European Atherosclerosis Society (EAS). Eur Heart J. 2011; 32(14): 1769-1818, doi: 10.1093/eurheartj/ehr158, indexed in Pubmed: 21712404.

11. Ponikowski P, Voors AA, Anker SD, et al. Authors/Task Force Members, Document Reviewers. 2016 ESC Guidelines for the diagnosis and treatment of acute and chronic heart failure: The Task Force for the diagnosis and treatment of acute and chronic heart failure of the European Society of Cardiology (ESC). Developed with the special contribution of the Heart Failure Association (HFA) of the ESC. Eur J Heart Fail. 2016; 18(8): 891-975, doi: 10.1002/ejhf.592, indexed in Pubmed: 27207191.

12. Rydén L, Grant PJ, Anker SD, et al. Authors/Task Force Members, ESC Committee for Practice Guidelines (CPG), Document Reviewers. ESC Guidelines on diabetes, pre-diabetes, and cardiovascular diseases developed in collaboration with the EASD: the Task Force on diabetes, pre-diabetes, and cardiovascular diseases of the European Society of Cardiology (ESC) and developed in collaboration with the European Association for the Study of Diabetes (EASD). Eur Heart J. 2013; 34(39): 3035-3087, doi: 10.1093/eurheartj/eht108, indexed in Pubmed: 23996285.

13. Williams B, Mancia G, Spiering W, et al. ESC Scientific Document Group. 2018 ESC/ESH Guidelines for the management of arterial hypertension. Eur Heart J. 2018; 39(33): 3021-3104, doi: 10.1093/eurheartj/ehy339, indexed in Pubmed: 30165516.

14. Montalescot G, Sechtem U, Achenbach S, et al. Task Force Members, ESC Committee for Practice Guidelines, Document Reviewers. 2013 ESC Guidelines on the management of stable coronary artery disease: the Task Force on the management of stable coronary artery disease of the European Society of Cardiology. Eur Heart J. 2013; 34(38): 2949-3003, doi: 10.1093/eurheartj/eht296, indexed in Pubmed: 23996286.

15. Siracuse JJ, Giles KA, Pomposelli FB, et al. Results for primary bypass versus primary angioplasty/stent for intermittent claudication due to superficial femoral artery occlusive disease. J Vasc Surg. 2012; 55(4): 1001-1007, doi: 10.1016/j.jvs.2011.10.128, indexed in Pubmed: 22301210.
16. Soga $\mathrm{Y}$, Iida $\mathrm{O}$, Hirano $\mathrm{K}$, et al. Mid-term clinical outcome and predictors of vessel patency after femoropopliteal stenting with self-expandable nitinol stent. J Vasc Surg. 2010; 52(3): 608-615, doi: 10.1016/j.jvs.2010.03.050, indexed in Pubmed: 20573476

17. Sobkowicz B, Tomaszuk-Kazberuk A, Kralisz P, et al. Coronary blood flow in patients with end-stage renal disease assessed by thrombolysis in myocardial infarction frame count method. Nephrol Dial Transplant. 2010; 25(3): 926-930, doi: 10.1093/ndt/gfp533, indexed in Pubmed: 19875377.

18. Ihnat DM, Duong ST, Taylor ZC, et al. Contemporary outcomes after superficial femoral artery angioplasty and stenting: the influence of TASC classification and runoff score. J Vasc Surg. 2008; 47(5): 967-974, doi: 10.1016/j.jvs.2007.12.050, indexed in Pubmed: 18372147.

19. Davies MG, Saad WE, Peden EK, et al. Percutaneous superficial femoral artery interventions for claudication-does runoff matter? Ann Vasc Surg. 2008; 22(6): 790-798, doi: 10.1016/j. avsg.2008.04.007, indexed in Pubmed: 18640817.

20. Wu TY, Giesler G, Herscu G, et al. Agreement among observers in the assignment of TransAtlantic Inter-Society Consensus classification and runoff score. J Vasc Surg. 2013; 58(5): 1254-1258, doi: 10.1016/j.jvs.2013.04.057, indexed in Pubmed: 23827336.

21. Akasaka T, Yoshida K, Kawamoto T, et al. Relation of phasic coronary flow velocity characteristics with TIMI perfusion grade and myocardial recovery after primary percutaneous transluminal coronary angioplasty and rescue stenting. Circulation. 2000; 101(20): 2361-2367, indexed in Pubmed: 10821811.

22. Kim AH, Shevitz AJ, Morrow KL, et al. Characterizing tissue perfusion after lower extremity intervention using two-dimensional color-coded digital subtraction angiography. J Vasc Surg. 2017; 66(5): 1464-1472, doi: 10.1016/j.jvs.2017.03.424, indexed in Pubmed: 28647197.

23. Salapura V, Blinc A, Kozak M, et al. Infrapopliteal run-off and the outcome of femoropopliteal percutaneous transluminal angioplasty. Vasa. 2010; 39(2): 159-168, doi: 10.1024/03011526/a000022, indexed in Pubmed: 20464672.

24. Prince MR, Chabra SG, Watts R, et al. Contrast material travel times in patients undergoing peripheral MR angiography. Radiology. 2002; 224(1): 55-61, doi: 10.1148/radiol.2241011338, indexed in Pubmed: 12091662.

Cite this article as: Kaczmarczyk P, Krzanowski M, Szybiak E, et al. Dynamics of below-the-knee arterial blood flow after endovascular revascularisation of peripheral arteries as a potential predictor of clinical outcomes during one-year follow-up. Kardiol Pol. 2019; 77(1): 24-32, doi: 10.5603/KP.a2018.0212.

\section{WHAT IS NEW?}

Several predictors of clinical outcomes after percutaneous interventions of the infrainguinal arteries have been demonstrated. The status of blood flow velocity in below-the-knee arteries after intervention assessed by angiography has not been determined. The aim of the study was to investigate whether the dynamics of blood flow in the below-the-knee arteries correlate with clinical outcomes after a 12-month follow-up period in patients with severe leg ischaemia treated percutaneously. After the procedure, blood flow in all patent below-the-knee arteries was assessed using frame count. During the 12-month follow-up, clinical condition, as well as reintervention and amputation rates were observed. Patients with higher frame count in the tibial anterior artery experienced significantly better clinical improvement within the follow-up period. Lower frame count predisposed to worse clinical outcomes after angioplasty. The results suggest better clinical outcomes during the follow-up in patients with slower blood flow assessed by frame count after endovascular interventions of the infrainguinal arteries. 